\title{
Clinical characteristics of anorectal Mycoplasma genitalium infection and microbial cure in men who have sex with men
}

\author{
Jason J. Ong $\mathrm{PhD}^{1,2,3,4}$, Ei Aung MBBS ${ }^{1}$, Tim R.H. Read PhD ${ }^{1,2}$, \\ Christopher K. Fairley $\mathrm{PhD}^{1,2}$, Suzanne M. Garland $\mathrm{PhD}^{5,6}$, Gerald Murray $\mathrm{PhD}^{5,7}$, \\ Marcus Y. Chen $\mathrm{PhD}^{1,2}$, Eric P.F. Chow $\mathrm{PhD}^{1,2}$, Catriona S. Bradshaw $\mathrm{PhD}^{1,2}$
}
${ }^{1}$ Melbourne Sexual Health Centre, Alfred Health, Carlton, Victoria, Australia
${ }^{2}$ Central Clinical School, Monash University, Melbourne, Victoria, Australia
${ }^{3}$ Department of General Practice, Faculty of Medicine, Dentistry and Health Sciences,
University of Melbourne, Victoria, Australia

${ }^{4}$ Department of Global Health and Development, London School of Hygiene and Tropical

Medicine, London, United Kingdom

${ }^{5}$ Centre for Women's Infectious Diseases, The Royal Women's Hospital, Parkville, Victoria,

Australia

${ }^{6}$ Department of Obstetrics and Gynaecology, University of Melbourne, Parkville, Victoria,

Australia

${ }^{7}$ Murdoch Childrens Research Institute, Parkville, Victoria, Australia

\section{Corresponding author:}

Jason J. Ong

580 Swanston Street

Carlton, Victoria 3053, Australia

Email: Jong@mshc.org.au 


\section{Conflicts of interest and source of funding}

Melbourne Sexual Health Centre has received research funding from SpeeDx Pty Ltd (Australia). This study was not funded by SpeeDx and no entity had input into its design or conduct. JJO, EPFC and TRHR are supported by the Australian National Health and Medical Research Council Early Career Fellowships (JJO: 1104781; EPFC: 1091226; TRHR: 1091536).

Short summary: A study of men who have sex with men with proctitis revealed that Mycoplasma genitalium (31\% [25-38]) was commonly detected, and microbiological cure with azithromycin was low (35\% [22-50]). 


\begin{abstract}

\section{Background}

We report clinical characteristics of proctitis caused solely by Mycoplasma genitalium(MG), compared with chlamydia and gonococcus. We determined the proportions cured with firstline (azithromycin) and second-line antimicrobials (moxifloxacin, pristinamycin).
\end{abstract}

\title{
Methods
}

166 patients attending Melbourne Sexual Health Centre from 2012 to 2016 with symptoms of proctitis were tested for MG, Chlamydia trachomatis and Neisseria gonorrhoeae. Demographic characteristics, sexual behaviours, clinical symptoms and signs were recorded. Multinomial multivariable logistic regression was used to test for significant differences in symptoms and signs for the pathogens detected.

\section{Results}

Seventeen percent of men had MG (95\% confidence interval [12-24]), 21\% chlamydia [1527], and 40\% gonococcal monoinfection [32-48], whilst 22\% had MG coinfection [16-29]. Relative to men with MG monoinfection, those with chlamydial monoinfection reported more anal pain (adjusted prevalence odds ratio(aPOR) 4.68, [1.41-14.19]), while men with gonococcal monoinfection reported more anal pain (aPOR 6.75, [2.21-20.55]) and tenesmus (aPOR 15.44, [1.62-146.90]), but less anal itch (aPOR 0.32, [0.11-0.93]). The microbiological cure for MG using azithromycin was low at 35\% [22-50], while moxifloxacin subsequently cured 92\% [64-100], and pristinamycin 79\% [54-94] of infections.

\section{Conclusion}

MG was almost as common as chlamydia in men presenting to a sexual health centre with symptoms of proctitis. Men with anorectal MG monoinfection were less likely to have symptoms and signs compared to those with chlamydia or gonococcus monoinfection. Cure for men with symptomatic anorectal MG by azithromycin was low. We suggest routine testing for MG in cases of proctitis, with test of cure following treatment being essential.

Key words: Mycoplasma genitalium, men who have sex with men, proctitis, Chlamydia trachomatis, Neisseria gonorrhoea 


\section{INTRODUCTION}

Mycoplasma genitalium (MG) is a sexually transmitted infection (STI), and has recently been implicated in lower and upper reproductive tract disease in women, and non-gonococcal urethritis in men $(1,2)$. However, less is known about its prevalence and contribution to anorectal symptoms in men who have sex with men (MSM). Melbourne Sexual Health Centre (MSHC) has reported a significant increase in MG positivity in anal swabs from MSM, from $2.5 \%$ in 2009 to $12.7 \%$ in 2014 (p-trend=0.005), which was not the case for urine and urethral samples(3). However, a limitation of that study was that the anal samples were from men regardless of symptoms, so it was not clear what proportion were from men who had symptoms of proctitis or from an asymptomatic contact of MG. A smaller MSHC study examining aetiological agents of proctitis between 2012 and 2013 that included 21\% HIV-positive men, reported that $12 \%$ of cases had detectable MG(4): however, the study did not provide information on the clinical presentation of $\mathrm{MG}$ proctitis compared to other aetiological agents. A Ugandan study reported the clinical presentation of urogenital MG may differ from other aetiological agents(5), but we are unaware of data on the clinical characteristics of MG-positive proctitis.

No STI guidelines recommend a treatment regimen specifically for MG-positive proctitis(68) with treatment based on current recommendations for urogenital MG infections(9). Increasing macrolide resistance for urogenital MG is reported and a recent meta-analysis of urogenital MG showed that the pooled cure for azithromycin in studies before 2009 was $85 \%$ (95\% confidence interval (CI):82-88) declining to $67 \%$ (95\% CI: $57-77)(10)$ in studies after 2009. Importantly, these data did not include anorectal MG and were largely studies of heterosexual men. In Melbourne, three consecutive studies reported declining cure rates for MG following azithromycin over the last decade: 85\% (95\% CI: 77-90) in 2005-2007(11), 69\% (95\% CI: 60-80) in 2008-2011(12), and 61\% (95\% CI: 53-69) in 2012-13(13). These studies were dominated by urogenital MG with very few anorectal cases. 
In this study, we report the clinical characteristics associated with proctitis in MSM attending MSHC between 2012 and 2016 in whom MG was detected and to compare this with the characteristics associated with the most established causes of proctitis, chlamydial and gonococcal proctitis. We also compared the group of men who tested positive to MG and had other coinfections to examine the effect of MG alone (i.e. whether there were differences between $\mathrm{Mg}$ monoinfection and $\mathrm{Mg}$ coinfection). We report the proportions cured with first line (azithromycin) and second-line antimicrobials (moxifloxacin, pristinamycin). To date, there has not been any published data for antimicrobials such as moxifloxacin and pristinamycin for management of anorectal MG, so we provide the first data for these two agents.

\section{METHODS:}

\section{Study participants}

We obtained routinely collected data from MSHC's electronic medical record database of patients diagnosed with proctitis who were tested for all three pathogens (Mycoplasma genitalium, Chlamydia trachomatis and Neisseria gonorrhoeae) and had at least one detected. We included MSM aged 18 years and over, who attended MSHC from June 2012 to May 2016. MSM status was self-reported at registration as someone who ever had any oral or anal sex with another man. To avoid duplication of detecting persistent infection, we excluded men who presented with proctitis who had had the same organism detected within the previous 6 months. All cases included in the study were single episodes of proctitis.

\section{Measurement}

Demographic data were collected including patient's age, HIV serostatus, whether they had a current regular partner, the number of casual partners in the last 3 and 12 months, and any condomless receptive anal sex within the last 3 and 12 months. Clinical data collected 
included patient symptoms, the duration (anal pain, anal discharge, anal bleeding, anal itch, tenesmus) and clinical signs (inguinal lymphadenopathy, anal bleeding, discharge and/or ulceration).

Proctitis was a clinical diagnosis made by the clinician, defined as anal pain with or without discharge and bleeding on examination. Proctoscopy was discretionary, performed in a subset of males $(36 \%)$ in keeping with clinic practice due to poor tolerability in symptomatic patients and with increasing use of highly sensitive nucleic acid amplification testing (NAAT) where direct visualization and sampling from inflamed mucosa is not required. From June 2012, clinic guidelines recommended all men who presented to MSHC with proctitis have anal swabs to test for $C$. trachomatis (CT), N. gonorrhoeae (NG), and $M$. genitalium. Testing for Treponema pallidum and herpes simplex virus (HSV) was performed at clinician's discretion. MG testing was performed at the Molecular Microbiology Laboratory at the Royal Women's Hospital using an in-house real-time polymerase chain reaction (PCR) assay targeting the 16s rRNA gene(14). Before March 2015, chlamydia was detected using strand displacement amplification (BD Probetec) and gonorrhoea was detected by culture on modified Thayer Martin medium. After March 2015, chlamydia and gonorrhoea were detected using the APTIMA Combo 2 assay (GenProbe Diagnostics)(15). All positive gonorrhoea results were confirmed using the Aptima GC test (Gen-Probe Diagnostics, San Diego, California)(16), which uses a different nucleic acid target. When chlamydia was detected, genotyping for LGV was performed using previously published methods with minor modifications(17). The equivalent serovar was identified by finding the closest matching nucleotide sequence of L2 and L2b from Genebank no. M14738 and AY586530. HSV detection was by PCR targeting the glycoprotein B gene using conventional PCR and a real-time version of this(18). Treponema pallidum was tested using a TaqMan real-time PCR assay(19). 


\section{Statistical analysis}

We stratified the demographic and sexual behaviour data by monoinfection with MG, chlamydia, or gonorrhoea, and coinfection of MG with other STIs (this included NG, CT, LGV, HSV and syphilis, and will henceforth be referred to as 'MG coinfection'). We excluded from further analysis, men who did not have rectal MG and had two or more other rectal STIs. To assess for statistically significant differences, we used Fisher's exact test for categorical data and Kruskal-Wallis test for non-parametric continuous data. We conducted a multinomial multivariable logistic regression to test for significant differences between specific clinical features of proctitis caused by different aetiological agents. The dependent variable was categorized into four different groups: MG monoinfection (reference group), chlamydial monoinfection, gonococcal monoinfection, and MG coinfection. This was adjusted for age, HIV status and condomless receptive anal sex in the last 3 months. Prevalence odds ratios were calculated with the level of statistical significance at $\mathrm{p}<0.05$. Successful treatment outcomes were identified as men who had a repeat negative test for MG at least two weeks after treatment with first line (azithromycin) or second line (moxifloxacin or pristinamycin) antimicrobials. Men were routinely recalled for their test of cure. All analyses were conducted using STATA software version 13.1 (StataCorp, College Station, TX, USA).

Ethical approval was obtained from the Alfred Human Research Ethics Committee (308/16).

\section{RESULTS}

\section{Study population}

During the study period, 201 MSM with 212 episodes of proctitis were tested for the three STIs: M. genitalium, C. trachomatis and N. gonorrhoeae. Cases were tested for anorectal herpes in $82 \%(174 / 212)$, anorectal syphilis using NAAT in $57 \%(121 / 212)$ and, of the men 
with anorectal chlamydia, 68\% (54/80) were tested for anorectal LGV. The most prevalent agent detected in cases with symptoms of proctitis was gonorrhoea $(54 \%, 95 \%$ confidence interval [47-61], followed by chlamydia (38\% [31-45]) and MG (31\% [25-38]). LGV was detected in $4 \%(2 / 54)$ of men whose positive chlamydia anal swab was also tested for LGV strains.

For further analysis describing clinical features of proctitis, we excluded cases who had two or more other rectal STIs but no $\operatorname{MG}(\mathrm{n}=46)$. Therefore, we retained for analysis monoinfection cases with MG (17\%, [12-24], 29/166), chlamydia (20\%, [15-27], 34/166), gonorrhoea (40\%, [32-48], 66/166), and MG coinfection (22\%, [16-29], 37/166). Men with MG coinfection had: herpes (38\%, [22-55], 14/37), gonorrhoea (27\%, [14-44], 10/37), chlamydia (27\%, [14-44], 10/37), syphilis (22\%, [10-38], 8/37), and LGV (5\%, [1-18], 2/37) detected. Seven men had three concurrent pathogens.

The demographic characteristics are summarized in Table 1. Men with Mg monoinfection had a higher median age (33 years), compared to those with $\mathrm{Mg}$ coinfection (29 years), chlamydial monoinfection (27 years) and gonococcal monoinfection $(25$ years $)(\mathrm{P}<0.001)$. Men with MG monoinfection had less condomless receptive anal sex within the last 3 months $(52 \%)$ compared with chlamydial monoinfection $(88 \%)$, gonococcal monoinfection $(71 \%)$, and $\mathrm{MG}$ coinfection $(59 \%)(\mathrm{P}=0.01)$. There was a higher proportion of $\mathrm{HIV}$ in men with $\mathrm{MG}$ monoinfection (28\%) and MG coinfection (35\%), compared with chlamydial monoinfection $(12 \%)$, and gonococcal monoinfection $(14 \%)(\mathrm{p}=0.05)$.

\section{Clinical characteristics of anorectal MG}

When adjusted for age, HIV status and condomless anal sex in the last 3 months, relative to men with MG monoinfection, men with chlamydial monoinfection were more likely to report 
anal pain (adjusted prevalence odds ratio (aPOR) 4.68 [1.41-14.19]), Table 2. Men with gonococcal monoinfection were more likely to report both anal pain (aPOR 6.75 [2.21-20.55]) and tenesmus (aPOR 15.44 [1.62-146.90]), but were less likely to report anal itch (aPOR 0.32 [0.11-0.93]). Men with MG coinfection were more likely to report anal pain (aPOR 4.45 [1.39-14.19]) and anal ulceration (RRR 4.27 [1.02-17.73]) compared to men with MG monoinfection; however $38 \%$ of MG coinfected cases had HSV.

\section{Treatment outcomes}

The majority of cases with MG returned for a test of cure $(77 \%, 51 / 66)$. All cases who returned for a test of cure received azithromycin first line and the overall proportion with microbiological cure was low at $35 \%$ (95\% CI:22-50, 18/51). Thirteen cases had moxifloxacin as a second-line antimicrobial with an overall microbiological cure proportion of 92\% (95\% CI:64-100, 12/13). Twenty-one cases received pristinamycin as a second-line antimicrobial with an overall microbiological cure of 79\% (95\% CI:54-94, 15/19).

\section{DISCUSSION}

This study of MSM attending a sexual health centre in Melbourne, Australia, investigated the clinical features of proctitis in which Mycoplasma genitalium was detected, and reported susceptibility to first- and second-line antimicrobials. It adds to the limited literature on anorectal MG-infection by reporting that anorectal MG was as common as chlamydia in MSM presenting with symptoms of proctitis. However, MG proctitis was associated with fewer symptoms, indicative of lesser clinical severity of inflammation compared to cases of chlamydia or gonorrhoea detected as the sole pathogen. Of concern but not unexpectedly, as with urethral infections the majority of MG-positive proctitis was not susceptible to macrolides. 
We contribute several important observations regarding anorectal MG in MSM. First, MG appears likely to be a causative agent of proctitis. It is possible that MG may be incidentally detected in men presenting with symptoms of proctitis as untreated urogenital MG may last for months or years(20). However, against incidental detection is the fact that we found a significant difference in the anorectal MG prevalence between men with asymptomatic MG infection attending MSHC in another study compared to men with symptomatic MG. In an ongoing study at our service, 401 asymptomatic MSM were tested for anorectal MG and MG was detected in 5.0\% (95\% CI: 3.1, 7.6)(21). In support of MG causing symptomatic anorectal infection, we found six-fold higher positivity of anorectal MG amongst cases presenting with proctitis, compared to this background prevalence in asymptomatic $\operatorname{MSM}(21)$.

Second, consistent with research in pelvic inflammatory disease(22), our findings suggests that MG may be less inflammatory compared to Chlamydia trachomatis and Neisseria gonorrhoea, indicating MG may be a more indolent infection in men and women(23). Previous literature has been conflicting regarding the association between MG and symptomatic anorectal infection. This may be partly influenced by patient selection and the health seeking patterns across populations. In a small study from USA of 27 MSM with anorectal MG, investigators reported that rectal symptoms (rectal pain, bleeding, discharge or tenesmus) were moderately associated with rectal MG detection (OR 2.8, 95\% CI: 1.08.0)(24). However, they did not report the duration of symptoms nor stratify their analysis according to specific symptoms. Conversely, in another small study from the UK of 19 MSM with anorectal MG, no associations were found between MG and symptoms of proctitis(25). This difference may either be explained by their small sample size or that bacterial load of MG may influence symptoms for both urethral and anorectal MG(4, 26). It is noteworthy that men presenting with symptomatic anorectal NG had higher bacterial loads compared to men with anorectal NG but without any rectal symptoms(27). 
Not unexpectedly, the majority of infections with MG proctitis were not susceptible to the first-line treatment of azithromycin. This is consistent with published evidence showing rising resistance in urogenital MG infections globally(28). Further, recent MSHC data in asymptomatic MSM confirms 90\% (95\% CI: 68, 99) of men with anorectal MG had macrolide resistance(21). The overall cure of symptomatic anorectal MG with subsequent antimicrobials (moxifloxacin, pristinamycin) was consistent with the cure rates of $88 \%$ cure reported for moxifloxacin and $75 \%$ cure reported for pristinamycin found in urogenital $\operatorname{MG}(13,29)$. To the best of our knowledge, this is the first published data on the use of these agents for anorectal MG infection. These data indicate similar overall efficacy of the three antimicrobials for anorectal infection, as is reported for urogenital infection, but highlight the need for use of combined diagnostic resistance assays to facilitate selection of appropriate antimicrobials at the time of diagnosis and routine test of cure(30).

The strength of the study includes using a relatively large database of MSM with symptoms of proctitis, who were also comprehensively tested for other STIs. The majority of men returned for a test of cure for MG, enabling us to report the proportion of microbiological cure in men receiving various antimicrobials. This study should be read in light of some limitations. First, we did not perform proctoscopy in the majority of cases, as this is an uncomfortable procedure of doubtful clinical value now that we have sensitive diagnostic tests that do not require direct visualization for collection. Second, gonococcal cases were predominantly culture positive and therefore likely to represent high bacterial load infections which may bias our results towards more symptoms being associated with anorectal GC. Finally, we do not have contemporaneous data on the prevalence of MG in asymptomatic controls, which would enable us to establish an association between MG and proctitis, although an association appears likely from the our recent asymptomatic prevalence study mentioned(21). 
In conclusion, current international STI guidelines do not have clear recommendations for testing and treatment of MG in men presenting with proctitis(6-8). This study found that MG was almost as common as chlamydia in men presenting to a sexual health centre with symptoms of proctitis but appears to be associated with less marked symptoms than chlamydia and gonorrhoea. These data are consistent with the evidence supporting the role of MG in PID, where it has taken many years to establish an association with PID. Symptom severity suggestive of anorectal inflammation appears to be very similar between the three STIs in PID and proctitis with MG seemingly the more indolent STI in both clinical syndromes. Based on the findings of this study we recommend: i) testing for MG in men presenting with symptoms of proctitis, ii) use of combined diagnostic-resistance assays to facilitate early selection of appropriate antimicrobials and iii) routine test of cure following antimicrobials. Cure following moxifloxacin and pristinamycin appears to be in line with that reported for urogenital MG. These data highlight the fact that MG is a relevant STI in MSM.

Author contribution: JJO, CB, and TRHR contributed to the conception and design of the study. JJO and EA collected the data. JJO analysed the data and drafted the paper. All authors revised the manuscript and approved the final version to be published. 


\section{REFERENCES}

1. McGowin CL, Anderson-Smits C. Mycoplasma genitalium: an emerging cause of sexually transmitted disease in women. PLoS Pathog. 2011;7(5):e1001324.

2. Lis R, Rowhani-Rahbar A, Manhart LE. Mycoplasma genitalium infection and female reproductive tract disease: a meta-analysis. Clin Infect Dis. 2015;61(3):418-26.

3. Tabrizi SN, Costa AM, Marceglia A, et al. Mycoplasma genitalium testing pattern and infection rates over a six year period in Melbourne, Australia The International Society for Sexually Transmitted Diseases Research 2016.

4. Bissessor M, Tabrizi SN, Bradshaw CS, et al. The contribution of Mycoplasma genitalium to the aetiology of sexually acquired infectious proctitis in men who have sex with men. Clin Microbiol Infect. 2016;22(3):260-5.

5. Vandepitte J, Bukenya J, Hughes $\mathrm{P}$, et al. Clinical characteristics associated with Mycoplasma genitalium infection among women at high risk of HIV and other STI in Uganda. Sex Transm Dis. 2012;39(6):487-91.

6. Centres for Disease Control and Prevention. 2015 Sexually transmitted diseases treatment guidelines. Accessed at http://www.cdc.gov/std/tg2015. Accessed 7th September 2016.

7. de Vries HJ, Zingoni A, White JA, Ross JD, Kreuter A. 2013 European Guideline on the management of proctitis, proctocolitis and enteritis caused by sexually transmissible pathogens. Int J STD AIDS. 2014;25(7):465-74.

8. British Association for Sexual Health and HIV Guidelines. Accessed at https://www.bashh.org/guidelines Accessed 17th October 2017.

9. Jensen JS, Cusini M, Gomberg M, Moi H. 2016 European guideline on Mycoplasma genitalium infections. J Eur Acad Dermatol Venereol. 2016;30(10):1650-6. 
10. Lau A, Bradshaw CS, Lewis D, et al. The Efficacy of Azithromycin for the Treatment of Genital Mycoplasma genitalium: A Systematic Review and Meta-analysis. Clin Infect Dis. 2015;61(9):1389-99.

11. Bradshaw CS, Jensen JS, Tabrizi SN, et al. Azithromycin failure in Mycoplasma genitalium urethritis. Emerg Infect Dis. 2006;12(7):1149-52.

12. Bradshaw CS, Chen MY, Fairley CK. Persistence of Mycoplasma genitalium following azithromycin therapy. PLoS One. 2008;3(11):e3618.

13. Bissessor M, Tabrizi SN, Twin J, et al. Macrolide resistance and azithromycin failure in a Mycoplasma genitalium-infected cohort and response of azithromycin failures to alternative antibiotic regimens. Clin Infect Dis. 2015;60(8):1228-36.

14. Twin J, Taylor N, Garland SM, et al. Comparison of two Mycoplasma genitalium real-time PCR detection methodologies. J Clin Microbiol. 2011;49(3):1140-2.

15. Bachmann LH, Johnson RE, Cheng H, et al. Nucleic acid amplification tests for diagnosis of Neisseria gonorrhoeae and Chlamydia trachomatis rectal infections. J Clin Microbiol. 2010;48(5):1827-32.

16. Smith DW, Tapsall JW, Lum G. Guidelines for the use and interpretation of nucleic acid detection tests for Neisseria gonorrhoeae in Australia: a position paper on behalf of the Public Health Laboratory Network. Commun Dis Intell Q Rep. 2005;29(4):35865.

17. Lister NA, Tabrizi SN, Fairley CK, Smith A, Janssen PH, Garland S. Variability of the Chlamydia trachomatis omp1 gene detected in samples from men tested in maleonly saunas in Melbourne, Australia. J Clin Microbiol. 2004;42(6):2596-601.

18. Druce J, Catton M, Chibo D, et al. Utility of a multiplex PCR assay for detecting herpesvirus DNA in clinical samples. J Clin Microbiol. 2002;40(5):1728-32.

19. Leslie DE, Azzato F, Karapanagiotidis T, Leydon J, Fyfe J. Development of a realtime PCR assay to detect Treponema pallidum in clinical specimens and assessment 
of the assay's performance by comparison with serological testing. J Clin Microbiol. 2007;45(1):93-6.

20. Vandepitte J, Weiss HA, Kyakuwa N, et al. Natural history of Mycoplasma genitalium infection in a cohort of female sex workers in Kampala, Uganda. Sex Transm Dis. 2013;40(5):422-7.

21. Read TRH, Fairley CK, Murray G, et al. Prevalence of Mycoplasma genitalium and macrolide resistance in asymptomatic men who have sex with men attending a sexual health centre. . Sexually Transmitted Infections;93(Suppl 2):A28.

22. Haggerty CL, Taylor BD. Mycoplasma genitalium: an emerging cause of pelvic inflammatory disease. Infect Dis Obstet Gynecol. 2011;2011:959816.

23. Short VL, Totten PA, Ness RB, Astete SG, Kelsey SF, Haggerty CL. Clinical presentation of Mycoplasma genitalium Infection versus Neisseria gonorrhoeae infection among women with pelvic inflammatory disease. Clin Infect Dis. 2009;48(1):41-7.

24. Francis SC, Kent CK, Klausner JD, et al. Prevalence of rectal Trichomonas vaginalis and Mycoplasma genitalium in male patients at the San Francisco STD clinic, 20052006. Sex Transm Dis. 2008;35(9):797-800.

25. Soni S, Alexander S, Verlander N, et al. The prevalence of urethral and rectal Mycoplasma genitalium and its associations in men who have sex with men attending a genitourinary medicine clinic. Sex Transm Infect. 2010;86(1):21-4.

26. Jensen JS, Bjornelius E, Dohn B, Lidbrink P. Use of TaqMan 5' nuclease real-time PCR for quantitative detection of Mycoplasma genitalium DNA in males with and without urethritis who were attendees at a sexually transmitted disease clinic. J Clin Microbiol. 2004;42(2):683-92.

27. Bissessor M, Tabrizi SN, Fairley CK, et al. Differing Neisseria gonorrhoeae bacterial loads in the pharynx and rectum in men who have sex with men: implications for 
gonococcal detection, transmission, and control. J Clin Microbiol. 2011;49(12):43046.

28. Unemo M, Jensen JS. Antimicrobial-resistant sexually transmitted infections: gonorrhoea and Mycoplasma genitalium. Nat Rev Urol. 2017;14(3):139-52.

29. Tim R.H. Read, Jørgen S. Jensen, Christopher K. Fairley, et al. Treatment outcomes and factors influencing response to pristinamycin for macrolide-resistant Mycoplasma genitalium. Emerg Infect Dis (in press).

30. Tabrizi SN, Tan LY, Walker S, et al. Multiplex Assay for Simultaneous Detection of Mycoplasma genitalium and Macrolide Resistance Using PlexZyme and PlexPrime Technology. PLoS One. 2016;11(6):e0156740. 
Table 1 Demographics of cases with MG monoinfection, chlamydial monoinfection, gonococcal monoinfection and MG coinfection,

Melbourne Sexual Health Centre (2012-2016), $N=166$.

\begin{tabular}{|c|c|c|c|c|c|}
\hline Variable & $\begin{array}{l}\text { MG monoinfection } \\
(N=29) \\
n(\%) \text { or median [IQR] }\end{array}$ & $\begin{array}{l}\text { Chlamydial } \\
\text { monoinfection }(N=34) \\
n(\%) \text { or median }[\mathrm{IQR}]\end{array}$ & $\begin{array}{l}\text { Gonococcal } \\
\text { monoinfection }(N=66) \\
n(\%) \text { or median }(I Q R)\end{array}$ & $\begin{array}{l}\text { MG coinfection } \\
(N=37) \\
n(\%) \text { or median [IQR] }\end{array}$ & $P$ value \\
\hline Age (years) & $33[28-38]$ & $27[24-33]$ & $25[22-31]$ & 29 [26-33] & $<0.001$ \\
\hline HIV-positive & $8(28)$ & $4(12)$ & $9(14)$ & $13(35)$ & 0.05 \\
\hline Had a current regular partner & $13(45)$ & $11(32)$ & $27(41)$ & $8(22)$ & 0.22 \\
\hline Number of casual partners in the last 12 months & $5[3-10]$ & $8[5-15]$ & $6[3-12]$ & $10[5-25]$ & 0.29 \\
\hline Number of casual partners in the last 3 months & $2[1-4]$ & $4[3-6]$ & $3[1-6]$ & $3[2-5]$ & 0.25 \\
\hline Condomless receptive anal sex in the last 3 months & $15(52)$ & $30(88)$ & $47(71)$ & $22(59)$ & 0.01 \\
\hline
\end{tabular}

IQR = interquartile range 
Table 2 Characteristics associated with proctitis in which MG was detected as the sole pathogen compared to chlamydial monoinfection,

\section{gonococcal monoinfection and MG coinfection, Melbourne Sexual Health Centre (2012-2016), N=166}

\begin{tabular}{|c|c|c|c|c|c|c|}
\hline Characteristic & $\begin{array}{r}\text { Chlamydial monoinfection } \\
\text { vs. MG monoinfection } \\
\text { Adjusted POR }{ }^{\#}(95 \% \mathrm{Cl})\end{array}$ & $P$ value & $\begin{array}{r}\text { Gonococcal monoinfection } \\
\text { vs. MG monoinfection } \\
\text { Adjusted POR }(95 \% \mathrm{Cl})\end{array}$ & $P$ value & $\begin{array}{r}\text { MG coinfection vs. } \\
\text { MG monoinfection } \\
\text { Adjusted POR }{ }^{\#}(95 \% \mathrm{Cl})\end{array}$ & $P$ value \\
\hline \multicolumn{7}{|l|}{ Symptoms } \\
\hline - Anal pain & $4.68(1.41-14.19)^{*}$ & 0.01 & $6.75(2.21-20.55)$ & $<0.01$ & $4.45(1.39-14.19) *$ & 0.01 \\
\hline - Anal pain duration (per additional day) & $1.00(0.99-1.02)$ & 0.62 & $0.97(0.93-1.01)$ & 0.13 & $0.99(0.98-1.01)$ & 0.48 \\
\hline - Anal discharge & $0.89(0.29-2.73)$ & 0.85 & $2.02(0.75-5.48)$ & 0.17 & $1.19(0.41-3.44)$ & 0.74 \\
\hline - Anal discharge duration (per additional day) & $1.00(0.96-1.03)$ & 0.79 & $0.96(0.90-1.02)$ & 0.17 & $0.94(0.85-1.03)$ & 0.17 \\
\hline - Anal bleeding & $1.07(0.35-3.31)$ & 0.91 & $0.71(0.25-2.03)$ & 0.52 & $0.64(0.21-2.00)$ & 0.45 \\
\hline - Anal bleeding duration (per additional day) & $0.99(0.98-1.01)$ & 0.52 & $0.93(0.87-1.00)$ & 0.06 & $0.92(0.83-1.03)$ & 0.15 \\
\hline - Anal itch & $0.52(0.16-1.64)$ & 0.26 & $0.32(0.11-0.93)^{*}$ & 0.04 & $0.50(0.17-1.50)$ & 0.22 \\
\hline - Anal itch duration (per additional day) & $1.09(0.92-1.29)$ & 0.31 & $0.81(0.60-1.07)$ & 0.14 & $1.09(0.93-1.29)$ & 0.30 \\
\hline - Tenesmus & $4.24(0.37-49.06)$ & 0.25 & $15.44(1.62-146.90)^{*}$ & 0.02 & $6.10(0.60-62.08)$ & 0.13 \\
\hline - Tenesmus duration (per additional day) & $0.77(0.43-1.38)$ & 0.38 & $1.13(0.73-1.73)$ & 0.59 & $0.88(0.55-1.41)$ & 0.60 \\
\hline \multicolumn{7}{|l|}{ Examination findings } \\
\hline - Anal discharge & $2.49(0.43-14.50)$ & 0.31 & $3.93(0.79-19.55)$ & 0.10 & $3.17(0.58-17.31)$ & 0.18 \\
\hline - Inguinal lymphadenopathy & - & - & - & - & - & - \\
\hline - Anal bleeding & - & - & - & - & - & \\
\hline - Anal ulcer & $0.49(0.07-3.35)$ & 0.47 & $0.69(0.15-3.28)$ & 0.65 & $4.27(1.02-17.73)^{*}$ & 0.04 \\
\hline
\end{tabular}

${ }^{\#}$ multinomial multivariate logistic regression analysis adjusted for age, HIV status and condomless receptive anal sex in the last 3 months; ${ }^{*} \mathrm{p}<0.05,{ }^{* *} \mathrm{p}<0.01 ;{ }^{5}$ Too few cases with variability for inguinal lymphadenopathy and anal bleeding to calculate an adjusted POR; POR= Prevalence odds ratio, $95 \% \mathrm{Cl}=95 \%$ confidence interval 